Renata Franco Pimentel Mendes ${ }^{1}$

SILVIO MARTINELU'

ROBERTO EDUARDO BitTAR²

Rossana Pulcinel Viera Francisco ${ }^{2}$

MARCELO ZUGAiB ${ }^{2}$

\title{
Fatores associados ao falso diagnóstico pré-natal da restrição de crescimento fetal
}

\author{
Factors associated with false diagnosis of fetal growth restriction
}

Artigo Original

Palavras-chave:
Retardo do crescimento fetal
Peso fetal
agnóstico obstétrico e ginecológico

Erros de diagnóstico

Ultrassonografia pré-natal

Testes de função placentária

Monitorização fetal

Keywords

Fetal growth retardation

Fetal weight

Diagnostic techniques, obstetrical and gynecological

Diagnostic errors

Ultrasonography, prenatal

Placental function tests

Fetal monitoring

\section{Resumo}

OBJETIVO: Descrever os fatores relacionados ao falso diagnóstico de restrição de crescimento fetal (RCF). MÉTODOS: Foram incluídas 48 gestantes encaminhadas ao nosso serviço com suspeita de RCF, não confirmada após o nascimento. Estas foram comparadas ao grupo de gestantes com RCF confirmada e foram descritas características relacionadas a esses falso-positivos. Os dados foram analisados utilizando-se o programa Statplus para Mac ${ }^{\circledR}$, versão 5.8. Os resultados obtidos no estudo foram divididos em variáveis categóricas e contínuas para análise. Para comparação entre proporções, foi aplicado o teste do $\chi^{2}$ ou o teste exato de Fisher. $\bigcirc$ nível de significância foi estabelecido em $\mathrm{p}<0,05$ para todos os testes. RESULTADOS: As gestantes com falso diagnóstico de restrição de crescimento fetal apresentavam as seguintes características: chegaram ao serviço em idade gestacional mais precoce (média de 32,8 semanas); entre 2 e 6 exames de ultrassonografia antes da matrícula no hospital terciário (média 3,8); foram submetidas à ultrasonografia até a $12^{a}$ semana em apenas $25 \%$ dos casos; tinham medida da altura uterina normal em 66,7\% dos casos; foram submetidas a pelo menos 1 ultrassonografia com percentil normal em 52,1\% dos casos; tinham a última ultrassonografia (média de 36 semanas) com percentil médio de 18; foram submetidas em média a 5 exames de ultrassonografia e 4,6 exames de vitalidade após ingressarem no serviço. CONCLUSÃO: $\bigcirc$ falso diagnóstico da RCF envolve custos hospitalares altos e demanda maior de especialistas. Deve-se valorizar a medida da altura uterina, por meio de exame físico cuidadoso e confirmar esse diagnóstico com a ultrassonografia nas últimas semanas de gestação, antes que a conduta obstétrica seja tomada.

\section{Abstract}

PURPOSE: The aim of this study was to analize and describe some characteristics related to a false diagnosis of intrauterine growth restriction (IUGR). METHODS: We retrospectively included 48 pregnant women referred to our service with a suspected diagnosis of IUGR that was not confirmed after birth and we compared them to another group with confirmed IUGR. We then analyzed the characteristics of the false-positive results. The results of the study were divided into continuous and categorical variables for analysis. The $\chi^{2}$ test or Fisher exact test was applied to compare proportions. The level of significance was set at $\mathrm{p}<0.05$ for all tests. RESULTS: In our sample, pregnant women with a false diagnosis of IUGR had the following characteristics: they were referred earlier (mean gestational age of 32.8 weeks); were submitted to 2 to 6 ultrasound examinations before been registered in our service; in $25 \%$ of cases ultrasound examination was performed before 12 weeks; in $66.7 \%$ of cases the symphysis-fundal height measurement was normal; in $52.1 \%$ of cases they had at least 1 sonographic exam above the $10^{\text {th }}$ percentile; on average, the last ultrasound examination (performed on average at 36 weeks) was above the $18^{\text {th }}$ percentile; the women were submitted to a mean number of 5 ultrasound examinations and to a mean number of 4.6 vitality exams. CONCLUSION: The false diagnosis of IUGR involves high hospital costs and higher demand for specialists. The symphysis-fundal height measurement must be valued, and the diagnosis of IUGR must be confirmed with ultrasonography in the last weeks of pregnancy before any obstetric management is taken.
Correspondêncio

Renata Franco Pimentel Mendes Avenida Doutor Eneas de Carvalho Aguiar, 255 - Cerqueira César (EP: 05403-010 São Paulo (SP), Brasil

Recebido $21 / 01 / 2014$

Aceito com modificacōoes $26 / 05 / 2014$
Departamento de Obstetrícia e Ginecologia da Faculdade de Medicina da Universidade de São Paulo - USP - São Paulo (SP), Brasil. Hospital das Clínicas, Faculdade de Medicina, Universidade de São Paulo - USP - São Paulo (SP), Brasil.

2Departamento de Obstetrícia e Ginecologia, Faculdade de Medicina, Universidade de São Paulo - USP - São Paulo (SP), Brasil. Conflito de interesses: nada a declarar. 


\section{Introdução}

Recém-nascidos portadores de restrição de crescimento fetal (RCF) apresentam maior frequência de complicações perinatais $^{1-3}$. Como o peso de nascimento é apenas uma medida final, antecipar o diagnóstico pode contribuir para a redução da morbimortalidade ${ }^{4-6}$.

A determinação exata da idade gestacional é imprescindível para o diagnóstico de RCF - sua confirmação só pode ser realizada após o nascimento. Durante a gestação, o diagnóstico provável de RCF é realizado por métodos clínicos e ultrassonográficos ${ }^{7,8}$.

Como método clínico, a altura uterina é a mais utilizada. A medida da altura uterina inferior ao percentil 10 para a idade gestacional constitui sinal clínico suspeito e deve ser sempre utilizada para o rastreamento de RCF. Apesar de ser método barato e de fácil acesso, a medida da altura uterina pode sofrer interferência de vários fatores como: índice de massa corpórea e paridade materna, volume de líquido amniótico e apresentação fetal ${ }^{9}$. Por esse motivo, deve ser complementada, nos casos suspeitos, pela ultrassonografia obstétrica, que representa nos dias atuais o principal instrumento de detecção dos desvios do crescimento fetal durante a gravidez $z^{10,11}$.

Considera-se a presença de RCF suspeita quando o peso estimado do feto pela ultrassonografia é inferior ao percentil 10 para a idade gestacional. Em virtude dos inúmeros fatores epidemiológicos que podem influenciar o peso fetal, a Organização Mundial da Saúde (OMS) recomenda que cada população tenha a sua própria curva de crescimento ${ }^{12,13}$. No entanto, nosso país carece de uma curva padrão de crescimento fetal. A curva mais utilizada na maioria dos serviços de excelência é a curva de Hadlock et al. ${ }^{14,15}$.

Diante da suspeita de RCF, preconizamos exames de ultrassonografia quinzenais, para monitorizar o crescimento fetal, e exames de vitalidade fetal semanais, a partir da $26^{a}$ semana, por meio da cardiotocografia, do perfil biofísico fetal e da dopplervelocimetria ${ }^{16-18}$. Tal procedimento visa à monitoração fetal e possibilita uma intervenção precoce, caso necessária ${ }^{19,20}$.

Embora a ausência do diagnóstico da RCF seja apontada como a principal causa de mau resultado na gestação, o falso diagnóstico também apresenta implicações impor$\operatorname{tantes}^{21,22}$. Envolve custos hospitalares altos, demanda maior de especialistas, além de aumentar o estresse e a ansiedade materna. Apesar de toda essa importância, muito pouco foi relatado na literatura sobre fatores que podem estar associados a esse falso diagnóstico. $\mathrm{O}$ conhecimento desses fatores poderia evitar condutas desnecessárias antes da confirmação final do déficit de crescimento do feto, que não trariam benefício ao produto conceptual, como exames seriados e até a antecipação do parto. Em nosso estudo, procuramos, em gestantes encaminhadas com diagnóstico suspeito de RCF e não confirmado após o nascimento, descrever algumas características relacionadas a esses falso-positivos.

\section{Métodos}

Trata-se de um estudo caso-controle não pareado realizado no Hospital das Clínicas da Faculdade de Medicina da Universidade de São Paulo (HCFMUSP) no período de 2006 a 2013. Este estudo foi aprovado pela Comissão de Ética para Análise de Projetos de Pesquisa do HCFMUSP.

Gestantes encaminhadas ao Setor de Baixo Peso Fetal com suspeita de RCF foram comparadas quanto ao peso de nascimento superior ou inferior ao percentil 10 para a idade gestacional. Foram incluídas gestações únicas com idade gestacional bem estabelecida, acima da $25^{a}$ semana, e fetos com ausência de malformações.

A idade gestacional foi estabelecida por meio de dois métodos: data da última menstruação (DUM) compatível com a idade gestacional determinada pela ultrassonografia obstétrica realizada até a $12^{\mathrm{a}}$ semana, ou pelo menos duas ultrassonografias com idades gestacionais concordantes até a $20^{\mathrm{a}}$ semana.

O parâmetro clínico utilizado foi a medida da altura uterina, realizada da sínfise púbica até o fundo uterino com fita métrica. O percentil de altura uterina foi calculado segundo curva-padrão do próprio serviço, a Martinelli et al. ${ }^{23,24}$. O peso fetal estimado pela ultrassonografia foi calculado pela fórmula de Hadlock et al. ${ }^{14}$, levando em conta medidas do diâmetro biparietal (DBP), circunferência cefálica (CC), circunferência abdominal (CA) e comprimento do fêmur (F). A curva utilizada para o diagnóstico pós-natal de RCF foi a de Alexander et al. ${ }^{25}$.

As gestantes foram divididas em dois grupos: grupo estudo e grupo controle. No grupo estudo (não RCF), foram incluídas 48 gestantes com peso de nascimento com percentil superior a 10 para a idade gestacional. E no grupo controle (RCF), foram incluídas 278 gestantes cujos pesos de nascimento eram inferiores ao percentil 10 para a idade gestacional.

Os dados foram obtidos do banco de dados de registro das pacientes do Ambulatório de Baixo Peso Fetal, por meio de software próprio (Microsoft Access $2007^{\circledR}$ ). Foram analisadas as seguintes variáveis: idade, paridade e idade gestacional na primeira consulta de pré-natal no setor; número de exames ultrassonográficos prévios à admissão; percentil de peso da primeira ultrassonografia no serviço; idade gestacional e percentil de peso da última ultrassonografia realizada; número de casos em que o percentil da altura uterina era superior a 10 na primeira consulta; e número de casos em que pelo menos um exame de ultrassonografia tinha percentil superior a 
10 - nesse subgrupo: a idade gestacional e o percentil do exame normal, idade gestacional, peso e percentil de nascimento e total de exames de ultrassonografia e de vitalidade realizados no serviço.

Os dados foram analisados utilizando-se o programa Statplus para Mac, versão $5.8^{\circledR}$. Os resultados obtidos no estudo foram divididos em variáveis categóricas e contínuas para análise. As variáveis categóricas foram analisadas descritivamente, calculando-se frequências absolutas e relativas. Para análise das variáveis contínuas, os resultados foram expressos em médias, medianas, desvios padrão, frequências absolutas e relativas. Para comparação entre proporções, foi aplicado o teste do $\chi^{2}$, e, quando pertinente, o teste exato de Fisher. O nível de significância foi estabelecido em $\mathrm{p}<0,05$ para todos os testes.

\section{Resultados}

As principais características sociodemográficas das gestantes incluídas estão apresentadas na Tabela 1 . Não houve diferença significante entre os grupos em relação a essas variáveis.

A idade gestacional da primeira consulta de pré-natal no grupo não RCF variou de 25,3 a 37,9 semanas, com média e desvio padrão de 32,8 2 ,9 semanas, respectivamente. No grupo RCF, a idade gestacional variou de 26,4 a 39,8 semanas (média de 34,3 semanas e desvio padrão de 2,6 semanas). Houve diferença significativa entre os grupos $(\mathrm{p}<0,001)$.

O percentil da primeira ultrassonografia realizada no serviço no grupo não RCF foi em média 10,7 99,0 , comparado ao do grupo RCF, de 7,7 $\pm 9,9(\mathrm{p}=0,02)$.

As gestantes encaminhadas ao Setor de Baixo Peso Fetal que tiveram recém-nascidos com peso superior ao percentil 10 (grupo não RCF) haviam sido avaliadas em mínimo de 2 e máximo de 6 exames de ultrassonografia previamente, com média de 3,8 e desvio padrão de 1,0. Ainda nesse grupo, dos 48 casos analisados, apenas 12 $(25 \%)$ tinham ultrassonografia de $1^{\circ}$ trimestre. As gestantes encaminhadas que tiveram recém-nascidos com peso inferior ao percentil 10 (grupo RCF) foram submetidas a no mínimo 1 exame ultrassonográfico prévio e no

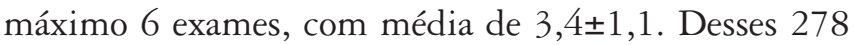

casos, $100(36 \%)$ foram submetidas à ultrassonografia de $1^{\circ}$ trimestre. Os grupos também se mostraram diferentes segundo esses critérios $(\mathrm{p}=0,007)$.

Os conjuntos também foram comparados quanto ao número de casos em que o percentil de altura uterina era superior a 10 na primeira consulta de pré-natal. No grupo não RCF, em 32 casos $(66,7 \%)$ o percentil da altura uterina diferiu do percentil da ultrassonografia na primeira consulta. No grupo RCF, em 133 casos (44,2\%) a altura uterina era superior ao percentil 10 para a idade gestacional.

O grupo não RCF apresentou 25 casos $(52,1 \%) \mathrm{em}$ que pelo menos 1 exame de ultrassonografia mostrava peso estimado do feto superior ao percentil 10 para a idade gestacional, e o grupo RCF, 83 casos (29,8\%), como demonstra a Figura 1. No subgrupo não RCF, a idade gestacional média do exame de ultrassonografia normal foi de 33,5 $\pm 4,3$ semanas (com variação de 25,1 a 39,7 semanas), e o percentil médio, de $21,9 \pm 9,5$ (com variação de 10,9 a 42,7). No subgrupo RCF, a média da idade gestacional em que foi realizada a ultrassonografia normal foi 31,5 semanas, com desvio padrão de 4,3 (variação de 25,1 a 39,9 semanas) e o percentil médio de 22,0, com desvio padrão de 13,3 (variação de 10,1 a 67,6), como mostra a Tabela 2. A idade gestacional, nesses subgrupos, mostrou diferença estatística significante $(\mathrm{p}=0,04)$, ao contrário do percentil $(\mathrm{p}=0,9)$.

Quanto à última ultrassonografia realizada, o grupo não RCF mostrou idade gestacional média de 36,0 $\pm 2,5$ semanas, e percentil médio de $18,2 \pm 13,5$. No grupo RCF, a média da idade gestacional foi de 35,5 $\pm 2,4$ semanas, e o percentil médio, de $6,8 \pm 7,4$. A idade gestacional não se mostrou diferente entre os grupos ( $\mathrm{p}=0,1)$, o que não ocorreu com o percentil da ultrassonografia $(\mathrm{p}<0,01)$.

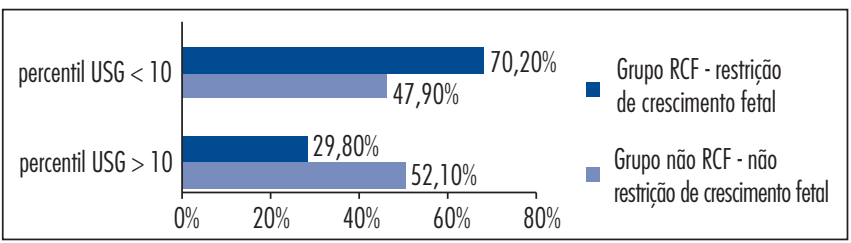

Figura 1. Distribuição das gestantes segundo a frequência de pelo menos uma ultrassonografia com peso fetal estimado acima do percentil 10

Tabela 1. Características sociodemográficas das gestantes

\begin{tabular}{|c|c|c|c|c|c|c|c|c|c|c|c|}
\hline \multirow{2}{*}{ Variáveis } & \multicolumn{5}{|c|}{ Grupo não RCF } & \multicolumn{5}{|c|}{ Grupo RCF } & \multirow{2}{*}{ Valor $\mathrm{p}$} \\
\hline & n & Média $\pm D P$ & Mediana & Mín. & Máx. & n & Média $\pm D P$ & Mediana & Mín. & Máx. & \\
\hline Idade & 48 & $28,4 \pm 5,7$ & 27,5 & 16 & 41 & 278 & $27,3 \pm 6,9$ & 26,5 & 15 & 42 & 0,5 \\
\hline $\begin{array}{l}\text { Número de } \\
\text { partos }\end{array}$ & 48 & $1,0 \pm 0,9$ & 1,0 & 0 & 3 & 278 & $0,9 \pm 1,0$ & 1,0 & 0 & 4 & 0,7 \\
\hline
\end{tabular}

Não RCF: não restrição de cerscimento fetal; RCF: restrição de crescimento fetal. 
Tabela 2. Médias, desvios padrão, medianas, mínimos e máximos dos resultados comparativos; comparação entre os grupos não RCF e RCF

\begin{tabular}{|c|c|c|c|c|c|c|c|c|c|c|c|}
\hline Variáveis & \multicolumn{5}{|c|}{ Grupo não RCF } & \multicolumn{5}{|c|}{ Grupo RCF } & Valor $p$ \\
\hline Idade gestacional da primeira consulta PN & 48 & $32,7 \pm 2,90$ & 33,5 & 25,3 & 37,9 & 278 & $34,3 \pm 2,6$ & 34,8 & 26,4 & 39,8 & 0,0002 \\
\hline Percentil de peso da primeira USG & 48 & $10,7 \pm 9,00$ & 8,5 & 3,0 & 43,0 & 278 & $7,7 \pm 9,9$ & 4,5 & 3,0 & 71,3 & 0,02 \\
\hline Número de exames USG prévios & 48 & $3,8 \pm 1,00$ & 4,0 & 2,0 & 6,0 & 278 & $3,3 \pm 1,0$ & 3,0 & 1,0 & 6,0 & 0,008 \\
\hline Idade gestacional da primeira USG normal & 25 & $33,5 \pm 4,30$ & 34,1 & 25,1 & 39,7 & 83 & $31,5 \pm 4,2$ & 31,3 & 25,4 & 39,9 & 0,049 \\
\hline Idade gestacional da última USG & 48 & $36,0 \pm 2,52$ & 36,5 & 27,7 & 39,7 & 278 & $35,5 \pm 2,4$ & 36,0 & 27,0 & 40,0 & 0,14 \\
\hline Percentil de peso da última USG & 48 & $18,2 \pm 13,50$ & 13,2 & 3,0 & 53,3 & 278 & $6,8 \pm 7,4$ & 4,2 & 3,0 & 67,6 & $<0,0001$ \\
\hline Número de exames de USG no serviço & 48 & $5,0 \pm 1,43$ & 5,0 & 2,0 & 9,0 & 278 & $4,6 \pm 1,5$ & 5,0 & 1,0 & 9,0 & 0,14 \\
\hline Número de exames de vitalidade no serviço & 48 & $4,6 \pm 2,60$ & 4,0 & 1,0 & 11,0 & 278 & $3,9 \pm 2,3$ & 4,0 & 1,0 & 12,0 & 0,21 \\
\hline
\end{tabular}

Não RCF: não restrição de cerscimento fetal; RCF: restrição de crescimento fetal; PN: pré-natal; USG: ultrassonografia.

O peso médio e o percentil médio de nascimento do grupo não RCF foram de 2994,7 g ( $\pm 355,5$ g) e 26,6 ( $\pm 17,7)$, respectivamente. No grupo RCF, a média do peso de nascimento foi de 2275,3 g ( $\pm 446,5 \mathrm{~g})$, e do percentil, de 5,5 $( \pm 1,1)$.

Após a matrícula da gestante no serviço, o grupo não RCF realizou em média 5,0 $\pm 1,4$ exames de ultrassonografia obstétrica, e o grupo RCF, uma média de 4,6 $6 \pm 1,5$ exames $(\mathrm{p}=0,14)$. Quanto aos exames de vitalidade realizados entre os casos do grupo não RCF após ingressar no serviço, estes variaram entre 1 e 11 (média de 4,6 exames), enquanto no grupo RCF o número de exames de vitalidade realizados variou de 1 a 12 (média de 3,9 exames), também sem diferença estatística $(\mathrm{p}=0,2)$.

\section{Discussão}

Esforços têm sido feitos com o objetivo de melhorar os métodos diagnósticos da restrição de crescimento fetal, visando à redução de sua morbimortalidade ${ }^{26-30}$. Em contraposição, há poucos estudos que mencionam efeitos adversos de resultados falso-positivos e fatores associados a esse falso diagnóstico. O objetivo do presente estudo foi apontar possíveis fatores associados ao diagnóstico falso-positivo de RCF no pré-natal.

Foi constatado o encaminhamento tardio das gestantes com suspeita de RCF ao Setor de Baixo Peso Fetal, uma vez que a idade gestacional média foi de 32,8 semanas no grupo de falso-positivos (não RCF) e de 34,3 semanas no grupo RCF. Além disso, o grupo não RCF foi submetido a maior número de ultrassonografias prévias à matrícula da gestante no serviço $(3,8 \times 3,4)$. Mesmo assim, isso não contribuiu significativamente para um diagnóstico acurado de RCF. Observamos também no presente estudo que no grupo não RCF apenas $1 / 4$ das pacientes havia sido avaliado com exame ultrassonográfico até a $12^{a}$ semana, em comparação ao grupo RCF, com $36 \%$ de ultrassonografias até essa fase. A idade gestacional, muitas vezes imprecisa, pode levar ao falso-positivo.
Quando comparado o percentil de peso da última ultrassonografia, este foi mais alto para o grupo não RCF. Portanto, em idade gestacional mais tardia, a caracterização do grupo de falso-positivos ficou mais acurada. No grupo não RCF, em 25 casos $(52,1 \%)$ verificou-se a presença de pelo menos 1 exame de ultrassonografia com percentil maior que 10 durante o seguimento no serviço, enquanto no grupo RCF isso ocorreu em 83 casos (29,8\%). Nesse subgrupo de pacientes, a idade gestacional do grupo não RCF foi mais tardia (33,5 $\times 31,5$ semanas) e apresentou diferença significante. O grupo de falso-positivos, portanto, apresentou maior probabilidade de ter pelo menos um exame ultrassonográfico superior ao percentil 10 até o parto ${ }^{31}$.

$\mathrm{Na}$ primeira consulta no Setor de Baixo Peso Fetal, em quase $67 \%$ dos casos do grupo de falso-positivos a medida da altura uterina na primeira consulta mostrou correlação com o peso de nascimento, e em $56 \%$ dos casos do grupo de RCF houve essa correlação. Um estudo que comparou a medida da altura uterina com a ultrassonografia mostrou sensibilidade de 76 e $83 \%$, respectivamente, para a predição da RCF no $3^{\circ}$ trimestre da gestação, mas sem demonstrar diferença estatística significativa ${ }^{32}$. Outro estudo, que avaliou a validade da estimativa do peso fetal por método baseado na altura uterina (regra de Johnson), não apresentou diferença do ponto de vista estatístico com a estimativa ultrassonográfica do cálculo do peso ao nascer ${ }^{33}$. Devemos valorizar, portanto, métodos clínicos em relação a esse diagnóstico e não considerar apenas a ultrassonografia, mesmo sendo o método mais importante . $^{34}$.

Nosso estudo aponta para alguns fatores relacionados ao diagnóstico incorreto de RCF. O impacto desse falso diagnóstico, porém, pode ser difícil de ser mensurado. No presente estudo, a realização do exame ultrassonográfico nas últimas semanas de gestação foi capaz de afastar o erro diagnóstico para a RCF, e a medida da altura uterina foi também importante para essa diferenciação. Métodos diagnósticos mais bem empregados podem otimizar recursos humanos e financeiros e contribuir para uma taxa menor de resultados falso-positivos. 
1. Mclntire DD, Bloom SL, Casey BM, Leveno KJ. Birth weight in relation to morbidity e mortality among newborn infants. $N$ Engl J Med. 1999;340(16):1234-8.

2. Piper JM, Xenakis EM, McFarland M, Elliott BD, Berkus $M D$, Langer $O$. Do growth-retarded premature infants have different rates of perinatal morbidity and mortality than appropriately grown premature infants? Obstet Gynecol. 1996; 87(2):169-74.

3. Wilcox AJ, Russell IT. Birth weight and perinatal mortality: II. On weight-specific mortality. Int J Epidemiol. 1983;12(3):319-25.

4. Chauhan S, Beydoun H, Chang E, Sandlin A, Dahlke JD, Igwe E, et al. Prenatal detection of fetal growth restriction in newborns classified as small for gestational age: correlates and risk of neonatal morbidity. Am J Perinatol. 2014;31(3):187-94.

5. American College of Obstetricians and Gynecologists. ACOG Practice bulletin no. 134: fetal growth restriction. Obstet Gynecol. 2013;121(5):1122-33.

6. Rocha CO, Bittar RE, Zugaib M. Neonatal outcomes of late-preterm birth associated or not with intrauterine growth restriction. Obstet Gynecol Int. 2010;2010:231842.

7. Rosenberg K, Grant JM, Hepburn M. Antenatal detection of growth retardation: actual practice in a large maternity hospital. Br J Obstet Gynecol. 1982;89(1):12-5.

8. Chauhan SP, Hendrix NW, Magann EF, Morrison JC, Kenney SP, Devoe LD. Limitations of clinical and sonographic estimates of birth weight: experience with 1034 parturients. Obstet Gynecol. 1998;91(1):72-7.

9. Curti A, Zanello M, De Maggio I, Moro E, Simonazzi G, Rizzo N, et al. Multivariable evaluation of term birth weight: a comparison between ultrasound biometry and symphysisfundal height. J Matern Fetal Neonatal Med. 2013 Nov 14. [Epub ahead of print]

10. Kayem G, Grangé G, Bréart G, Goffinet F. Comparison of fundal height measurement and sonographically measured fetal abdominal circumference in the prediction of high and low birth weight at term. Ultrasound Obstet Gynecol. 2009;34(5):566-71

11. Nahum GG, Stanislaw H. Ultrasonographic prediction of term birth weight: how accurate is it? Am J Obstet Gynecol. 2003; 188(2):566-74.

12. Nahum GG, Stanislaw H. Validation of a birth weight prediction equation based on maternal characteristics. J Reprod Med. 2002;47(9):752-60.

13. Gardosi J, Francis A. Controlled trial of fundal height measurement plotted on customised antenatal growth charts. Br J Obstet Gynaecol. 1999; 106(4):309-17.

14. Hadlock FP, Harrist RB, Martinez-Poyer J. In utero analysis of fetal growth: a sonographic weight standard. Radiology. $1991 ; 181(1): 129-33$.

15. Freire DMC, Cecatti JG, Paiva CSM. [Correlation between estimated fetal weight by ultrasound and neonatal weight]. Rev Bras Ginecol Obstet. 2010;32(1):4-10. Portuguese.

16. Zugaib M, Bittar RE. Protocolos assistenciais Clínica Obstétrica FMUSP. 4a ed. São Paulo: Atheneu; 2012.
17. Alfirevic Z, Stampalija T, Gyte GM. Fetal and umbilical Doppler ultrasound in high-risk pregnancies. Cochrane Database Syst Rev. 2010;(8):CD007529.

18. Society for Maternal-Fetal Medicine Publications Committee, Berkley E, Chauhan SP, Abuhamad A. Doppler assessment of the fetus with intrauterine growth restriction. Am J Obstet Gynecol. 2012;206(4):300-8.

19. Unterscheider J, Daly S, Geary MP, Kennelly MM, McAuliffe FM, $\mathrm{O}^{\prime}$ Donoghue K, et al. Definition and management of fetal growth restriction: a survey of contemporary attitudes. Eur J Obstet Gynecol Reprod Biol. 2014;174:41-5.

20. Royal College of Obstetricians and Gynaecologists. The investigation and management of the small-for-gestational-age fetus. 2 nd ed. London: RCOG; 2013.

21. Gruenwald P. Chronic fetal distress and placental insufficiency. Biol Neonat. 1963;5:215-65.

22. Miller HC, Hassanein K. Diagnosis of impaired fetal growth in newborn infants. Pediatrics. 1971;48(4):51 1-22.

23. Martinelli S, Bittar RE, Zuagib M. [Proposal of a new uterine height growth curve for pregnancies between 20 and 42 weeks]. Rev Bras Ginecol Obstet. 2001 ;23(4):235-41. Portuguese.

24. Martinelli S, Bittar RE, Zugaib M. [Prediction of fetal growth restriction by measurement of uterine height]. Rev Bras Ginecol Obstet. 2004;26(5):383-9. Portuguese.

25. Alexander GR, Himes JH, Kaufman RB, Mor J, Kogan M. A United States national reference for fetal growth. Obstet Gynecol. 1996;87(2): 163-8.

26. Ott WJ. Intrauterine growth retardation and preterm delivery. Am J Obstet Gynecol. 1993;168(6 Pt 1):1710-5.

27. Paz I, Gale R, Laor A, Danon YL, Stevenson DK, Seidman DS. The cognitive outcome of full-term small for gestational age infants at late adolescence. Obstet Gynecol. 1995;85(3):452-6.

28. Resnik R. Intrauterine growth restriction. Obstet Gynecol. 2002;99(3):490-6.

29. American College of Obstetricians and Gynecologists. Intrauterine growth restriction. Washington (DC): ACOG; 2000.

30. Chauhan SP, Dahlke JD, Magann EF, Chang E, Gupta L, Mol BW, et al. Isolated intrauterine growth restriction: a survey of Central Association of Obstetricians \& Gynecologists (CAOG) members. J Matern Fetal Neonatal Med. 2013;26(5):497-502.

31. Unterscheider J, Daly S, Geary MP, Kennelly MM, McAuliffe FM, $\mathrm{O}^{\prime}$ Donoghue K, et al. Optimizing the definition of intrauterine growth restriction: the multicenter prospective PORTO Study. Am J Obstet Gynecol. 2013;208(4):290.e 1-6.

32. Pearce JM, Campbell S. A comparison of symphysis-fundal height and ultrasound as screening tests for light-for-gestational age infants. Br J Obstet Gynaecol. 1987;94(2):100-4.

33. Cury AF, Garcia SAL. [Estimation of fetal weight: comparison between a clinical method and ultrasonography]. Rev Bras Ginecol Obstet. 1998;20(10):551-5. Portuguese.

34. Barker ED, McAuliffe FM, Alderdice F, Unterscheider J, Daly S, Geary MP, et al. The role of growth trajectories in classifying fetal growth restriction. Obstet Gynecol. 2013;122 (2 Pt 1):248-54. 